\title{
Mini review \\ Varicocele and male infertility: Historical, anatomical and urological perspectives - a mini review
}

\author{
Kalpana Ramachandran ${ }^{1}$, Sriram Krishnamoorthy ${ }^{2}$ \\ ${ }^{1}$ Professor and Head, Department of Anatomy, ${ }^{2}$ Professor, Department of Urology, Sri Ramachandra Institute of Higher \\ Education \& Research (SRIHER), Chennai 600116 Tamil Nadu, India
}

(Received: January $2021 \quad$ Revised: July $2021 \quad$ Accepted: July 2021)

Corresponding author: Kalpana Ramachandran.Email: kalpanasriram1@gmail.com

\begin{abstract}
Varicocele is defined as an abnormal dilatation of the pampiniform plexus of the spermatic cord. This is seen in 10 to $20 \%$ of general population and is one of the most common correctable causes of male infertility. The history of varicocele dates back to the era of Ancient Greeks to about $600 \mathrm{BC}$, who had sculptured varicocele in their statues as a tortuous bag of worms in the scrotum. It was Ambrose Pare who gave a poetic description to the pooling of blood in the spermatic cords and subsequently Curling coined the term Varicocele The intricacy of the venous drainage of the scrotum and its contents and the criss crossing of the veins makes the thermo-regulatory mechanism regulating spermatogenesis, even more complex. The Cremaster and Dartos greatly aid in facilitating the thermoregulatory mechanism of scrotal wall, which forms the anatomical basis of oligospermia in patients with varicocele. Apart from a visible change in seminal parameters, the benefits of varicocelectomy have extended on to an improvement in serum testosterone and enhancement of erectile and ejaculatory functions. With a greater knowledge of the pathophysiology of varicocele and with better understanding of the roles of gene polymorphisms and reactive oxygen species, the indications for varicocelectomy gain more support and evidence. A sound anatomical knowledge and an in-depth understanding of the events that take place at molecular level might shift the treatment focus in future from varicocelectomy to molecular targeted therapies.
\end{abstract}

Keywords: Varicocele; oligospermia; infertility; spermatozoa; pampiniform plexus.

\section{BACKGROUND}

$\mathrm{V}$ aricocele is defined as an abnormal dilatation of the pampiniform plexus of the spermatic cord (that surrounds the testicles) and dilatation of the internal spermatic vein (1). The dilated pampiniform plexus of veins of the scrotum resulting in oligospermia is one of the common and most important identifiable causes of male infertility (2). Various studies have shown a widespread prevalence of clinically detectable varicocele in 10 to $20 \%$ of the general population. This overall incidence doubles in infertile males and is seen in 25 to $40 \%$ of those with primary infertility and up to $80 \%$ of males with secondary infertility (3). As varicocele can result in a duration-dependent insult to the testicular function and to various indices of spermatozoa including morphology, progressive motility, density and vitality, a thorough understanding of the anatomical and urological aspects of varicocele become even more imperative (4). Multiple reasons are attributed to the alterations observed in seminal parameters secondary to varicocele. Thermoregulatory disturbances in the testicles, venous pooling, testicular hypoxia, refluxing renal and adrenal metabolites, higher levels of spermatozoa related reactive oxygen radicals are assumed to be the various factors that result in oligospermia (5). The objective of this review is to discuss the evolution of concepts of varicocele from historical, anatomical, and urological perspectives.

\section{Historical aspects}

The ancient Egyptian medical literatures that extensively describe surgical procedures like hernia and hydrocele do not mention about varicocele as a surgically correctable lesion (6). However, the portrayal of testicles can be traced back to $600 \mathrm{BC}$, where the Greek sculptures were depicted to have unequal sized testicles of varying heights with a few tortuous veins (7). It was then speculated that varicocele more commonly occurred in Greeks, as most people spent most of the daytime on their feet.

The earliest classical medical writing on varicocele was in $25 \mathrm{BC}$ to $50 \mathrm{AD}$, when Cornelius Celsus, a Roman Nobleman described two conditions that involved the dilatation of scrotal veins, namely "cirsocele" that caused dilation of deep veins and 'varicocele' that causes dilation of surface veins (8). However, it was Ambrose Pare in 1541, who gave a poetic definition of varicocele, calling that as 'compact blood vessels with stagnated melancholic blood and often seen in depressed men' (9). Three centuries later, Curling in 1853 coined the term 'varicocele' (dilatation of pampiniform plexus of veins) and related varicocele to oligospermia and male infertility (10).

The history of concepts of varicocele occurrence and its treatment is complex and has evolved over time. With conclusive evidence that this entity involves dilatation of pampiniform plexus including the 
testicular veins, varicocele as the most typical cause of primary infertility garnered support from all corners. William Tulloch in 1951 was credited with the first-ever surgery performed in patients with varicocele for treatment of infertility (11). His Robb's technique, where he approached the spermatic veins $5 \mathrm{~cm}$ above the internal inguinal ring, gained worldwide acceptance (12). Further modifications in procedures and advent of microsurgical techniques over the next few decades greatly revolutionized the outcomes of varicocelectomy for primary infertility.

\section{Anatomical aspects}

The anatomical arrangement designed by nature to protect and preserve the testicular function and sperm production may appear very simple to look at from outside butis too intricate to understand. Alsaikhan reports that the aetiology of varicocele is multifactorial, and the epidemiology and pathogenesis of this entity is still incompletely understood (13). A well-descended testicle is supported by two important and distinct muscular structures that have a definite role to play. The spermatic cord is surrounded by the Cremaster muscle that aids in drawing up the testicle in times of sudden fright towards the superficial inguinal ring. This defense mechanism is more prominent in the paediatric age than in adults. Of the tri-laminar venous drainage of testicles, the cremasteric vessels have been identified for long as the causative factor for the occurrence of varicocele. Hanley et al., in their 10-year-study on 450 infertile males concluded that in many patients, a varicocele is produced by tortuosity or varicosity of the cremasteric veins. Subsequently, many authors have concluded that the testicular veins were uninvolved in a majority and ligation of cremasteric veins was effective in relieving pain and improving fertility status (14).

The other muscular structure that supports the testicles and bears its entire weight during resting periods is the Dartos muscle (15). Dartos is extremely sensitive to changes in temperatures. By contracting or relaxing, the testicle can be moved towards or away from the superficial ring, thereby protecting the testicles from temperature variations, thus optimizing the temperature for adequate sperm production. These two muscular structures play a pivotal role in thermo-regulation of the scrotal contents. The pathophysiology of varicocele is centered on this phenomenon that leads to oligospermia and subsequent male infertility.

The complexity of venous drainage of the scrotum and its contents makes infertility in varicocele a little too complicated to comprehend. There are three layers of intercommunicating veins that drain blood from scrotum by forming the spermatic plexus of veins, also known as pampiniform plexus. These include the internal spermatic (testicular) veins that drain directly into the left renal vein or inferior vena cava on the right side. The deferential vein parallels the ductus deferens and empties into superior or inferior vesical veins. The cremasteric group of veins (external spermatic veins) drains into the inferior epigastric vein. Extensive inter-venous communications exist between the three veins. Another study documented a collateral venous flow in the scrotum and inguinal regions between the pampiniform plexus, cremasteric vessels and the veins overlying the various coverings of the cord. Comhaire postulated that the cross-over of venous channels existed through a sub-pubic anastomosis.

Varicocele more commonly affects the left testicle than the right. Several hypotheses have been postulated attributing to the commoner occurrence of left-sided Varicocele. In his review on the myths of varicocele and male infertility, Kantartzireporteda left-sided predominance in up to 78 to $93 \%$ of cases. A loaded sigmoid colon directly compressing on the left testicular vein, left testicular vein draining perpendicular to the left renal vein, left renal vein getting sandwiched between the abdominal aorta and superior mesenteric artery (Nutcracker syndrome), high levels of adrenal metabolites causing vasospasms of left renal vein are a few reasons for left-sided predominance (16).

The variations in the incidence of varicocele on both sides are also determined by the differences in internal spermatic veins (ISV). As the left spermatic vein drains perpendicular into the left renal vein and as the course of left ISV is longer than that of the right side, a higher pressure on the flow of blood from left spermatic veins is recorded. As long as the valves in the ISV are intact, a retrograde flow of blood is prevented. Absent or defective valves result in blood pooling, causing an increase in the intraluminal pressure and subsequent varicocele formation. However, various researchers have had a mixed opinion on the role of valves in varicocele formation. Braedel, in his study on venography in 659 patients with varicocele, observed that $73 \%$ of them did not show any demonstrable valves (17). They concluded that the disturbances that happen in secondary venous system development during embryogenesis might lead to idiopathic left-sided varicocele. Similarly, an autopsy study was done by Wishahi in 1991 on 70 human cadavers, who demonstrated an absence of valves in the spermatic veins and a cross-communication between the right and left spermatic veins, thereby questioning the reflux due to valvular incompetency to be a cause for varicocele. Retro-aortic renal veins causing posterior nutcracker syndrome can also lead on to varicoceles (18).

It may be envisaged, therefore, that a sound knowledge of fundamental anatomy and pathogenesis of this condition is an essential pre-requisite for a 
better understanding of the mechanism that leads on to varicocele induced male infertility.

\section{Urologist's perspectives}

The advent of Colour Doppler ultrasound has greatly revolutionized the diagnosis and staging of varicocele. Fig 1 illustrates the Doppler ultrasound of the scrotal vessels from patients with different grades of varicocele, but the four images do not necessarily depict the radiological criteria of the four grades of varicocele.

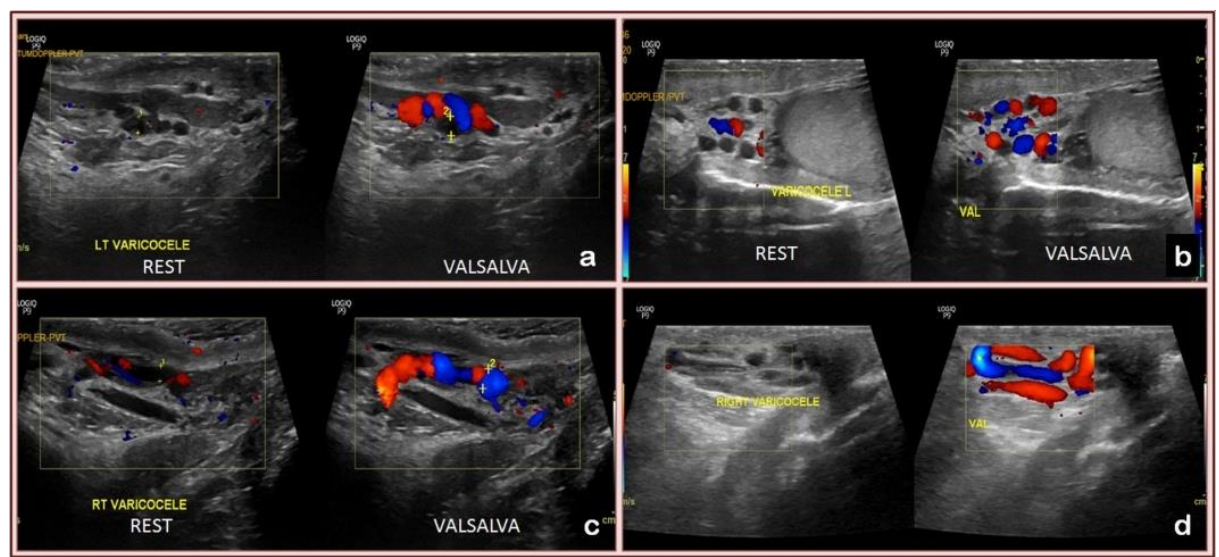

Fig 1: Doppler ultrasound of the scrotal vessels taken from patients with different grades of varicoceles. Subclinical (a), grade I (b), grade II (c) and grade III (d) varicoceles. (Four images do not necessarily fulfill the sonographic criteria of the 4 grades of varicocele).

Despite varicocele being considered to be one of the commonest surgically correctable causes of male infertility, the polemics surrounding the role of surgery in varicocele continue to daunt the treating urologists. Lack of plausible mechanisms to explain the reason for oligospermia or infertility in such patients and non-availability of meta-analyses to objectively document an improvement in seminal parameters after any particular type of varicocelectomy has resulted in controversies floating around the benefits of surgical intervention for varicocele.

Evers and Collins, in their systematic review of seven studies over 23-year period, concluded that varicocelectomy is not to be considered effective in treating males with primary infertility. The pregnancy rates in the treated couples' group and the control arms were almost similar. This study raises doubt about the exact role of varicocelectomy in infertility, as the pathogenic mechanisms in Varicocele appear multi-dimensional. Though various published reports highlight the benefits of varicocelectomy in improving oligospermia, the rationale for varicocelectomy in sub fertile males gathered tremendous momentum when its essential role extended beyond improving seminal parameters alone. In a retrospective review of 272 men who underwent microsurgical varicocelectomy, Kohn observed that microsurgical varicocelectomy resulted in a significant increase in absolute sperm count, sperm concentration and serum testosterone levels in all age groups, including men above 40 years of age (19). Najari et al., observed that varicocelectomy not only improves testosterone but also improves patientreported erectile and ejaculatory functions (20). However, meta-analysis by Baazeem et al., has categorically confirmed the beneficial effects of varicocelectomy in improvements in seminal parameters.

To eliminate such uncertainties from the minds of treating urologists, the American Society of Reproductive Medicine (ASRM) practice committee guideline has formulated the indications for treatment of Varicocele. Those couples wanting to conceive and who are known to have male-related factors, clinically grade 2 or 3 , and normal female partners are considered as ideal candidates for varicocelectomy.

Non-obstructive azoospermia (NOAZ) is another entity that may coexist with varicocele. Ever since Tulloch in 1952 reported the efficacy of varicocelectomy, numerous reports of improvements in seminal parameters and pregnancy rates in patients with NOAZ after varicocele repair have been published in the literature. Thakur, in his retrospective study in 104 patients on the role of infertility in NOAZ, reported that after varicocelectomy, $18 \%$ of them had sperms in their ejaculate. Though $10 \%$ of them showed spontaneous pregnancy, fertilization rate was $89 \%$ through viable sperms enough for performing assisted ICSI without TESE (21).

While controversies keep surrounding the indications for varicocelectomy, with an aim to obtain sufficient understanding of the condition, researchers further explore the pathophysiology of varicocele. The roles played by raised levels of reactive oxygen species (ROS) and oligospermia resulting from oxidative stress has been researched in great detail. The roles of gene polymorphisms of glutathione-S-transferase playing a role in influencing the surgical outcome of 
varicocelectomy in infertile population is also being studied (22). The active part of Heat Shock Proteins (HSP) and Heat Shock Factors (HSF) in causing varicocele-induced oligospermia is also being investigated. Ferlin, in his study on 117 patients with Varicocele observed that various HSP-A4 and HSFs 1 and 2 were expressed in the sperm of men with Varicocele and oligospermia and that these levels were over-expressed when the two entities occur together (23). Nixon observed that HSP A2 correlated well with maturation and function of sperms. An abnormal, dysfunctional expression of this protein resulted in oligoteratozoospermia (24).

\section{CONCLUSION}

The concept of evaluation and treatment of men with varicocele and primary male infertility has undergone a paradigm change. The evaluation of varicocele has progressed from clinical to the molecular level, with multiple molecular factors that have been identified to play a significant role in sperm quality and fertility status in men with varicocele. As most men with varicocele do not manifest with damaging effects on their fertility potential, it is convincing that not all patients with varicocele need surgical intervention. Hence, it is essential to know the overall incidence and prevalence of varicocele amongst normal and infertile males, which would greatly facilitate an estimation of the actual impact of this disease in sub fertile males.

With knowledge on the pathophysiology of varicocele increasing day by day, future treatments might aim at targeted molecular therapies rather than surgical intervention. Although further studies and translational researches are needed in this field of molecular biology, a thorough anatomical knowledge and detailed understanding of this clinical condition would greatly help us select the ideal candidate for varicocelectomy or provide targeted molecular therapies.

In this era of litigations and medico-legal cases and evidence-based practice methods, it is prudent to adopt further research on varicocele and follow the guidelines to identify who needs treatment, when to initiate treatment and what type of treatment is required to validate the rationale for varicocelectomy.

\section{CONFLICTS OF INTEREST}

Authors declare no conflict of interest.

\section{REFERENCES}

1. Lomboy, J. R., Coward, R. M. The Varicocele: Clinical presentation, evaluation, and surgical management. SeminInterventRadiol. 2016 Sep; 33(3): 163-169.

2. Chiba K., Ramasamy, R., Lamb, D. J., Lipshultz, L. I. The varicocele: diagnostic dilemmas, therapeutic challenges and future perspectives. Asian J Androl. 2016 Mar-Apr; 18(2): 276-281.

3. Smits, R. M., Mackenzie-Proctor, R., Yazdani, A., Stankiewicz, M. T., Jordan, V., Showell, M. G. Antioxidants for male subfertility. Cochrane Database Syst Rev. 2019 Mar 14; 3(3): CD007411.

4. Pastuszak, A. W., Wang, R. Varicocele and testicular function. Asian J Androl. 2015; 17(4): 659-667.

5. Steiner, A. Z., Hansen, K. R., Barnhart, K. T., Cedars, M. I., Legro, R. S., Diamond, M. P., et al., The effect of antioxidants on male factor infertility: the males, antioxidants, and infertility (MOXI) randomized clinical trial. Fertility and Sterility. 2020 Mar 1; 113(3): 552-560.

6. Marte, A. The history of varicocele: from antiquity to the modern ERA. IntBraz J Urol. 2018 May-Jun; 44(3): 563576.

7. Bonafini, B., Pozzilli, P. Scrotal asymmetry, varicocoele and the Riace Bronzes. Int J Androl. 2012 Apr; 35(2): 181-182.

8. Noske, H. D., Weidner, W. Varicocele-a historical perspective. World J Urol. 1999; 17: 151-157.

9. Shen, J. T., Weinstein, M., Beekley, A., Yeo, C., Cowan, S. AmbroiseParé (1510 to 1590): a surgeon centuries ahead of his time. Am Surg. 2014; 80: 536-538.

10. Eisenberg, M. L., Lipshultz, L. I. Varicocele-induced infertility: newer insights into its pathophysiology. Indian J Urol. 2011; 27(1): 58-64.

11. Tulloch, W. S. Consideration of sterility; subfertility in the male. Edinburg Med J. 1952; (59): 29-34.

12. Robb, W. A. Operative treatment of Varicocele. Br Med J. 1955; 2: 355-356.

13. Alsaikhan, B., Alrabeeah, K., Delouya, G. Epidemiology of Varicocele. Asian J of Andrology. 2016; 18(2): 179.

14. Jensen, C. F. S., Ostergren, P., Dupree, J. M., Ohl, D. A., Sønksen, J., Fode, M. Varicocele and male infertility. Nat Rev Urol. 2017 Sep; 14(9): 523-533.

15. Paick, S., Choi, W. S. Varicocele and testicular pain: A review. World J Mens Health. 2019 Jan; 37(1): 4-11.

16. Miro, I., Serrano, A., Pérez-Ardavín, J., March, J. A., Polo, A., Conca, M. Á., et al., Eighteen years of experience with pediatric nutcracker syndrome: the importance of the conservative approach. J Pediatr Urol. 2020 Apr; 16(2): 218. e1-218.e6.

17. Keene, D. J. B., Cervellione, R. M. Antegrade sclerotherapy in adolescent varicocele patients. J Pediatr Urol. 2017 Jun; 13(3): 305. e1-305.e6.

18. Lizama, L., Bran, S., Kuestermann, S., García-Gallont, R. Renal vein thrombosis due to posterior nutcracker syndrome. Methodist Debakey Cardiovasc J. 2020; 16(4): e5-e7.

19. Kohn, T. P., Kohn, J. R., Pastuszak, A. W. Varicocelectomy before assisted reproductive technology: are outcomes improved? Fertil Steril. 2017 Sep; 108(3): 385-391.

20. Najari, B. B., Introna, L., Paduch, D. A. Improvements in Patient-reported Sexual Function After Microsurgical Varicocelectomy. Urology. 2017 Dec; 110: 104-109.

21. Thakur, A. P., Sadasivan, D., Sharma, V., Ramasamy V, Parol S, Singh S, et al., Role of microsurgical varicocelectomy in the management of non-obstructive azoospermia with varicocele: our tertiary care centre experience. African Journal of Urology. 2020 Dec; 26(1): 18.

22. Ammar, O., Tekeya, O., Hannachi, I., Sallem, A., Haouas, Z., Mehdi, M. Increased Sperm DNA Fragmentation in Infertile Men with Varicocele: Relationship with Apoptosis, Seminal Oxidative Stress, and Spermatic Parameters. Reprod Sci. 2021 Mar; 28(3): 909-919.

23. Ning, J. Z., Rao, T., Cheng, F., Yu, W. M., Ruan Y, Yuan R, et al., Effect of varicocelectomy treatment on spermatogenesis and apoptosis via the induction of heat shock protein 70 in varicocele-induced rats. Mol Med Rep. 2017 Oct; 16(4): 5406-5412.

24. Nixon, B., Bromfield, E. G., Cui, J., De Iuliis, G. N. Heat shock protein A2 (HSPA2): Regulatory roles in germ cell development and sperm function. Adv Anat Embryol Cell Biol. 2017; 222: 67-93. 